\title{
Macroscopic Gas-Liquid Interfacial Equation Based on Thermodynamic and Mathematical Approaches
}

\author{
Yukihiro Yonemoto ${ }^{1}$ and Tomoaki Kunugi ${ }^{2}$ \\ ${ }^{1}$ Japan Atomic Energy Agency \\ ${ }^{2}$ Kyoto University \\ Japan
}

\section{Introduction}

At a gas-liquid interface, many complicated phenomena such as evaporation, condensation, electrokinesis, and heat and mass transfer occur. These phenomena are widely seen in various industrial and chemical systems. In chemical or biochemical reactive operations, bubble columns are used for increasing the mass transfer through the interface and for enhancing the separation of mixtures by rectification and water purification (Hong and Brauer 1989; Álvarez et al. 2000). However, the interfacial phenomena have various time and space scales (multi-scale) that are interrelated at the interface. Therefore, modeling gasliquid interfaces over a wide range of scales spanning molecular motion to vortical fluid motion is very difficult, and this has remained one of the key unresolved issues in multiphase flow science and engineering since a long time. In particular, the mechanism for bubble coalescence/repulsion behaviour is unknown, although it is a superficially simple behaviour and fundamental phenomena in bubbly flows. In order to evaluate the interfacial interactions such as bubble coalescence and repulsion quantitatively, we need a new gasliquid interfacial model based on the multi-scale concept which is expressed mathematically and that takes into account physical and chemical phenomena and heat and mass transfer at the interface.

In the theoretical point of view, the interfacial equation for a macroscopic-scale gas-liquid interface is mainly characterized by a jump condition. The macroscopic interface is discontinuous, and its physical properties such as density, viscosity, and temperature have discontinuous values. The jump condition has been discussed in terms of the mechanical energy balance (Scriven, 1960; Delhaye, 1974) using Stokes' theorem, the Gauss divergence theorem, differential geometry and so on. In these theorems, a test volume is considered at the interface between two continuous phases. In the derivation, the surface force acting on the discontinuous interface is modeled using the Young-Laplace equation. However, in such a mechanical approach, the definition of the curvature is unclear at the interface, and the surface tension coefficient is treated as a macroscopic experimental value. The interfacial model, which is based only on the mechanical energy balance, cannot take into account detailed physical and chemical phenomena occurring at the interface. In particular, the contamination at the interface, which is related to electric charges, is important for an 
interfacial interaction. Craig (2004) and Henry and Craig (2008) have discussed the effects of specific ions on bubble interactions. They have reported that bubble coalescence is affected by the ions adsorbed at the interface, combination of these ions, and the electrolyte concentration. Others have similarly reported the importance of electrolytes in bubble coalescence (Marčelja, 2004; Ribeiro \& Mewes, 2007; Tsang et al., 2004; Lessaard \& Zieminski, 1971). In our experimental research on microbubble flow (Yonemoto et al., 2008), some patterns have been observed with respect to microbubble coalescence. Microbubble coalescence has been estimated analytically using the film thinning theory. Results have shown that microbubble coalescence cannot be explained based only on hydrodynamics. That is to say, our results indicate the importance of mass transfer, which is related to contamination at the interface, for interfacial interaction.

Recently, the phase field theory (Cahn \& Hilliard, 1958) and van der Waals theory (Rowlinson \& Widom, 1984), wherein the interface is assumed to be a diffuse interface with a finite thickness, have been applied to perform numerical research on multiphase flow. The surface force is evaluated by the free energy defined at the interface and depends on both concentration (or density) and its gradient. Anderson et al. (1998) have reviewed the diffuseinterface models of hydrodynamics and their application to various interfacial phenomena. In their study, the diffuse-interface model was associated with the sharp-interface model (Delhaye, 1974). But the multi-scale concept was not expressed in concrete terms.

In a previous study (Yonemoto \& Kunugi, 2010a), a thermodynamic and mathematical interfacial model that takes into account the multi-scale concept has been developed on the basis of the phase field theory (Cahn \& Hilliard, 1958). In this model, we assumed that the interface has a finite thickness and that free energy is defined at the interface. In particular, the free energy is derived on a microscopic scale (Hamiltonian); this includes the electrostatic potential due to contamination at the interface. The free energy is incorporated into the Navier-Stokes (NS) equation by using the Chapman-Enskog expansion (Chapman \& Cowling, 1970), which mathematically discriminates the time and space scales of the interfacial phenomena. Finally, a new equation governing the fluid motion, called the multiscale multiphase flow equation, is derived. The multi-scale multiphase flow equation has been proven to have the potential to simulate interfacial interactions (Yonemoto \& Kunugi, $2010 \mathrm{~b})$. In the simulation, microbubble interaction is simulated and a liquid film between them is observed when the bubbles interact with each other. In the present study, the multiscale multiphase flow equation, which is the mesoscopic interfacial equation, was further discussed, and a macroscopic interfacial equation was derived based on our interfacial model. In particular, an interfacial jump condition treated by thermodynamics was derived from the multi-scale multiphase flow equation, and the thermodynamic interfacial jump condition was then compared with the conventional jump condition. In addition, we derived the Kelvin equation based on both the multi-scale multiphase flow equation (Yonemoto \& Kunugi, 2010a) and the thermodynamic jump condition. The present results indicate that our interfacial model can theoretically support various interfacial phenomena characterized by thermodynamics from a multi-scale viewpoint (micro to macro).

\section{Multi-scale multiphase flow equation}

In a previous study (Yonemoto \& Kunugi, 2010a), the multi-scale multiphase flow equation was derived based on the phase field theory (Cahn \& Hilliard, 1958). In the derivation, we assumed that the interface has a finite thickness similar to that of a fluid membrane 
(mesoscopic interface). The concept of the gas-liquid interface is shown in Fig. 1. Experimental observations of bubble interactions (Craig, 2004; Henry \& Craig, 2008) revealed that contamination at the interface may be an important factor. With this in mind, the contamination at the interface was associated with an electrostatic potential due to an electric double layer at the gas-liquid interface, and the free-energy equation, including the electrostatic potential, was then derived from a lattice-gas model (Safran, 1994):

$$
F=\int_{V}\left[f_{0}(\psi)+\frac{d}{2}(\nabla \psi)^{2}\right] \mathrm{d} V
$$

Here,

$$
\begin{gathered}
f_{0}(\psi)=-\frac{a}{2} \psi^{2}+\frac{b}{4} \psi^{4}-c z_{i} e V_{e} \psi \\
a=\frac{U_{\text {sum }}-4 k_{B} T}{l^{3}} \\
b=\frac{16 k_{B} T}{3 l^{3}} \\
c=\frac{1}{2 l^{3}} \\
d=\frac{U_{\text {sum }}}{2 l^{3}}
\end{gathered}
$$

The first and the second terms on the right-hand side of Eq. (1) denote the free energy in homogeneous and inhomogeneous systems, respectively. Coefficients $a\left[\mathrm{~J} / \mathrm{m}^{3}\right], b\left[\mathrm{~J} / \mathrm{m}^{3}\right], c$ $\left[1 / \mathrm{m}^{3}\right]$, and $d[\mathrm{~J} / \mathrm{m}]$ in Eqs. (1) and (2) have constant values. In Eqs. (3) and (6), $U_{\text {sum }}$ is related to an intermolecular potential. These values include microscopic physical information (Yonemoto \& Kunugi, 2010a). The symbols of $z_{\mathrm{i}}[-], e[\mathrm{C}], V_{\mathrm{e}}[\mathrm{V}]$, and $\psi$ [-] are the charge number, elementary charge, electrostatic potential, and order parameter, respectively. The third term on the right-hand side of Eq. (2) is related to the contamination at the interface. In general, the surface tension is evaluated by the surface tension coefficient and the curvature characterizing the macroscopic shape of the interface. As mentioned earlier, in our model, the interface has a finite thickness similar to that of a fluid membrane (Fig. 1). For an interface with finite thickness, it is very difficult to determine the geometric shape of the interface. Therefore, the surface tension should be considered from the free energy at the interface rather than from the curvature (Fialkowski et at., 2000; Yonemoto et al., 2005). Moreover, there are many physical and chemical processes at the interface that are characterized by various time and space scales. Therefore, in order to consider the various scale interactions that may arise among interfacial phenomena, the Chapman-Enskog expansion (Chapman \& Cowling, 1970) was applied to the NS equation. Here, the original NS equation is expressed as

$$
\rho \frac{\mathrm{D} \boldsymbol{u}}{\mathrm{D} t}=-\nabla \cdot T+\rho g
$$


where $\rho\left[\mathrm{kg} / \mathrm{m}^{3}\right], \boldsymbol{u}[\mathrm{m} / \mathrm{s}], \boldsymbol{T}\left[\mathrm{N} / \mathrm{m}^{2}\right], \boldsymbol{g}\left[\mathrm{m} / \mathrm{s}^{2}\right]$, and $t[\mathrm{~s}]$ represent the fluid density, velocity, stress tensor, acceleration due to gravity, and time, respectively.

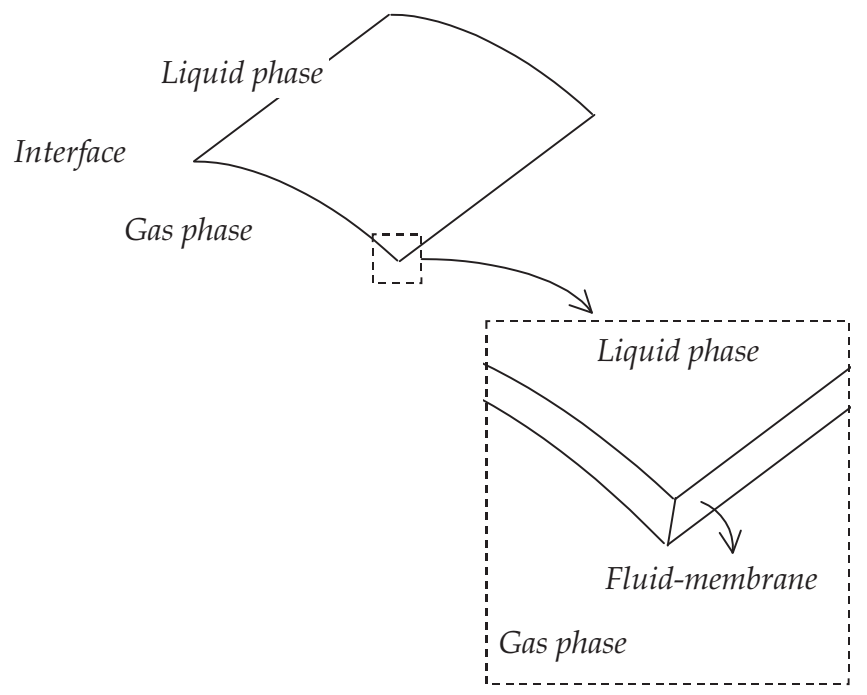

Fig. 1. Concept of a gas-liquid interface: macroscopic and mesoscopic image of the interface

Stress tensor $\boldsymbol{T}\left[\mathrm{N} / \mathrm{m}^{3}\right]$ is defined as $\boldsymbol{T}=P \boldsymbol{I}-\boldsymbol{\tau}$. The shear stress is $\tau\left[\mathrm{N} / \mathrm{m}^{2}\right]$. Pressure $P\left[\mathrm{~N} / \mathrm{m}^{2}\right]$ is the mechanical pressure, hereafter represented by $P_{\text {mech }}$. At this point, the operators $\mathrm{D} / \mathrm{D} t$ and $\nabla$ must include the various time and space scales (multi-scale concept). Therefore, in order to discriminate their scales, the Chapman-Enskog expansion was applied to $\mathrm{D} / \mathrm{D} t$ and $\nabla$ in the NS equation. The operators $\mathrm{D} / \mathrm{D} t$ and $\nabla$ were decomposed into the following expressions by using the small parameter $\varepsilon$ :

$$
\begin{gathered}
\boldsymbol{\nabla}=\boldsymbol{\nabla}^{(0)}+\varepsilon \boldsymbol{\nabla}^{(1)}+\varepsilon^{2} \nabla^{(2)}+\cdots+\varepsilon^{k} \nabla^{(k)}+\cdots \\
\frac{\mathrm{D}}{\mathrm{D} t}=\frac{\mathrm{D}}{\mathrm{D} t^{(0)}}+\varepsilon \frac{\mathrm{D}}{\mathrm{D} t^{(1)}}+\varepsilon^{2} \frac{\mathrm{D}}{\mathrm{D} t^{(2)}}+\cdots+\varepsilon^{k} \frac{\mathrm{D}}{\mathrm{D} t^{(k)}}+\cdots
\end{gathered}
$$

Here, superscript $k(k=0,1,2 \ldots)$ represents the scale of the phenomena, which becomes smaller as $(k)$ increases. For example, the superscript $(0)$ corresponds to the macroscopic scale. In Eqs. (8) and (9), the small parameter $\varepsilon$ is defined as $\varepsilon=\delta / L$. The symbols of $\delta$ $[\mathrm{m}]$ and $L[\mathrm{~m}]$ represent the characteristic lengths of the interface and the vortical fluid flow, respectively. After substituting Eqs. (8) and (9) into Eq. (7) and performing a simple tensor analysis, we obtain a new governing equation:

$$
\rho \frac{\mathrm{D} \boldsymbol{u}}{\mathrm{D} t^{(0)}}+\varepsilon \rho \frac{\mathrm{D} \boldsymbol{u}^{\prime}}{\mathrm{D} t^{(1)}}=-\nabla^{(0)} \cdot \boldsymbol{T}+\varepsilon \boldsymbol{\nabla}^{(1)} \cdot\left(f_{0}(\psi) \boldsymbol{I}\right)-\varepsilon d \boldsymbol{\nabla}^{(1)} \psi\left(\boldsymbol{\nabla}^{(1)} \cdot \nabla^{(1)} \psi\right)+\rho \boldsymbol{g}
$$

In the derivation, the free energy (Eq. (1)) is associated with thermodynamic pressure using the Maxwell relation. This equation is the multi-scale multiphase flow equation (Yonemoto \& Kunugi, 2010a). 


\section{Jump condition at gas-liquid interface}

\subsection{Momentum jump condition}

An interfacial phenomenon is complex and interpreted as a discontinuous problem. The interface separates two continuous equilibrium phases. When the curvature radius is considerably larger than the thickness of the interface, the equilibrium force balance at the interface is given by the following equation based on the interfacial coordinates shown in Fig. 2:

$$
\dot{\boldsymbol{M}}_{\mathrm{G}}+\dot{\boldsymbol{M}}_{\mathrm{L}}-\left\{\left(-P_{\mathrm{G}}\right) \boldsymbol{n}_{\mathrm{G}}+\boldsymbol{\tau}_{\mathrm{G}} \cdot \boldsymbol{n}_{\mathrm{G}}\right\}-\left\{\left(-P_{\mathrm{L}}\right) \boldsymbol{n}_{\mathrm{L}}+\boldsymbol{\tau}_{\mathrm{L}} \cdot \boldsymbol{n}_{\mathrm{L}}\right\}-2 H \sigma \boldsymbol{n}_{\mathrm{G}}-\frac{\mathrm{d} \sigma}{\mathrm{d} s} \boldsymbol{t}=0
$$

\section{Liquid phase}
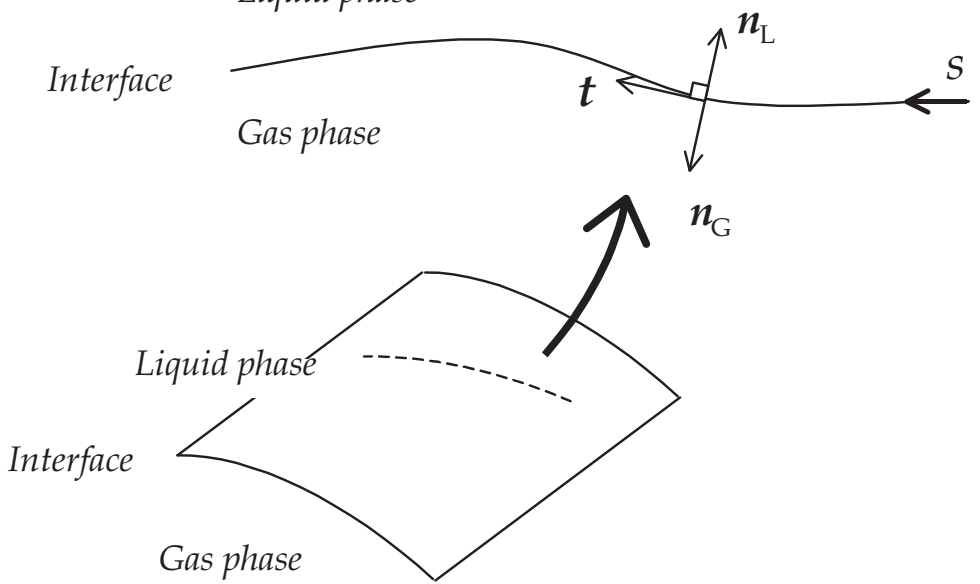

Fig. 2. Interfacial coordinates at the gas-liquid interface

where the subscripts $\mathrm{L}$ and $\mathrm{G}$ represent liquid and gas phases, respectively. This is called the momentum jump condition at the interface. The symbols $\sigma[\mathrm{N} / \mathrm{m}], P_{k}(\mathrm{k}=\mathrm{G}, \mathrm{L})\left[\mathrm{N} / \mathrm{m}^{2}\right]$, and $s[\mathrm{~m}]$ are the surface tension coefficient, pressure, and coordinate along the interface, respectively. The mean curvature is denoted by the symbol $H$; here, $H=\left(\kappa_{1}+\kappa_{2}\right) / 2$, where $\kappa_{1}$ and $\kappa_{2}[1 / \mathrm{m}]$ are the principal curvatures. The bold symbols $\boldsymbol{n}_{k}, \boldsymbol{t}$, and $\boldsymbol{\tau}_{k}\left[\mathrm{~N} / \mathrm{m}^{2}\right]$ are the unit normal, unit tangential vector, and shear stress, respectively. $\boldsymbol{M}_{k}[\mathrm{~kg} / \mathrm{ms}]\left(\dot{\boldsymbol{M}}_{k}\right.$ $\left[\mathrm{N} / \mathrm{m}^{2}\right]$ is the time derivative of $\boldsymbol{M}_{k}$ ) denotes the term related to mass transfer through the interface. In this paper, we call this equation the conventional jump condition. The jump condition at the interface is characterized by the curvature related to the shape of the interface, which means that the interface is a mathematical interface with zero thickness. Therefore, we consider the jump condition to be a macroscopic interfacial equation.

\subsection{Derivation of thermodynamic jump condition}

Here, the interfacial jump condition treated by thermodynamics is derived using the multi-scale multiphase flow equation (Eq. (10)). We call this derived condition the "thermodynamic jump condition" because Equation (10) is based on the thermodynamic concept (Yonemoto \& Kunugi, 2010a). 

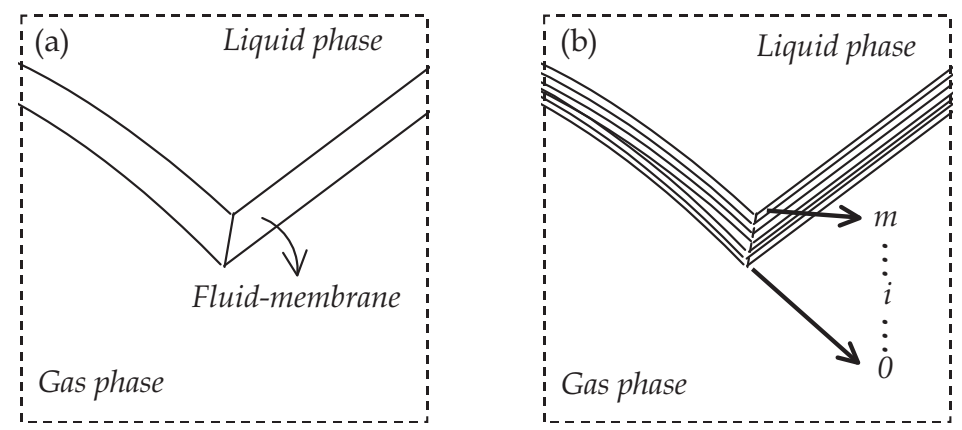

Fig. 3. Fluid membrane comprising elemental interfaces: (a) Fluid membrane, (b) Elemental interfaces. Interpretation of the fluid membrane based on interfacial coordinates

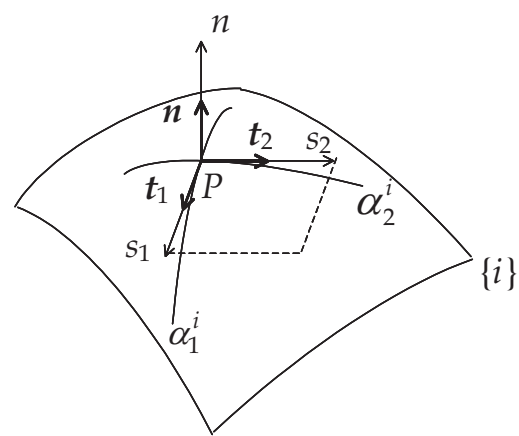

Fig. 4. Elemental interface $\{i\}$ with interfacial coordinates in a fluid membrane

The interfacial jump condition is mainly considered in the moving coordinate system. This means that this jump condition is discussed under the condition that the relative velocity between the convected interface and fluid motion that convects the interface is neglected. In particular, assuming that the interface is in the steady state, the left-hand side of Eq. (10) (substantial derivative term) is neglected. In this study, phase changes such as evaporation and condensation and the effect of gravity on the interface are not considered. Therefore, Equation (10) reduces to

$$
0=-\nabla^{(0)} \cdot \boldsymbol{T}+\varepsilon \boldsymbol{\nabla}^{(1)} \cdot\left(f_{0}(\psi) \boldsymbol{I}\right)-\varepsilon d \boldsymbol{\nabla}^{(1)} \psi\left(\boldsymbol{\nabla}^{(1)} \cdot \boldsymbol{\nabla}^{(1)} \psi\right) .
$$

Because Equation (11) is based on the interfacial coordinate system, Equation (12) is also transformed into an equation based on the interfacial coordinate system.

Before the concrete derivation of the thermodynamic jump condition, a mathematical operation is prepared. First, a test interface is defined as shown in Fig. 3. The test interface is divided into many elemental interfaces along the normal coordinate perpendicular to each elemental interface. We assume that the curvature is constant along the normal coordinate with respect to all elemental interfaces. Figure 4 shows representative interface $\{i\}$ for a number of elemental interfaces. Next, the interfacial coordinate system is considered at point $\mathrm{P}$ on its interface. The tangential axes are represented by $s_{1}$ and $s_{2}$, and $n$ is the normal 
axis. Unit vectors $\boldsymbol{t}_{1}, \boldsymbol{t}_{2}$, and $\boldsymbol{n}_{k}(k=\mathrm{G}$ or $\mathrm{L})$ are defined on the $s_{1}, s_{2}$, and $n$ axes, respectively. The unit vectors are orthogonal to each other. The direction of $\boldsymbol{n}_{\mathrm{L}}$ is upward, and $\boldsymbol{n}_{\mathrm{G}}$ is opposite to $\boldsymbol{n}_{\mathrm{L}}$ (i.e., $\boldsymbol{n}_{\mathrm{L}}=-\boldsymbol{n}_{\mathrm{G}}=\boldsymbol{t}_{1} \times \boldsymbol{t}_{2}$ ). In the present study, we focused on the local interface; therefore, general covariance (Aris, 1962) such as the change in the tangential vector along the interface is not considered.

Because $u_{i}=\left(s_{1}, s_{2}, n\right)$ and $x_{i}=(x, y, z)$, the operator $\nabla$ is transformed into the interfacial coordinate system, which leads to

$$
\boldsymbol{\nabla}=\boldsymbol{t}_{1} \frac{\partial}{\partial s_{1}}+\boldsymbol{t}_{2} \frac{\partial}{\partial s_{2}}+\boldsymbol{n} \frac{\partial}{\partial n}
$$

Thus, the operator $\boldsymbol{\nabla} \cdot \boldsymbol{\nabla}$ becomes

$$
\boldsymbol{\nabla} \cdot \boldsymbol{\nabla}=\left(\kappa_{1}+\kappa_{2}\right) \frac{\partial}{\partial n}+\frac{\partial^{2}}{\partial s_{1}^{2}}+\frac{\partial^{2}}{\partial s_{2}^{2}}+\frac{\partial^{2}}{\partial n^{2}}
$$

where $\kappa_{1}$ and $\kappa_{2}$ are the principal curvatures at the interface. In what follows, for the sake of simplicity, only the $s_{1}-n$ coordinate system is considered. Using Eqs. (13) and (14), we can rewrite Eq. (12) as

$$
\begin{aligned}
0 & =-\boldsymbol{t}_{1} \frac{\partial P_{\text {mech }}}{\partial s_{1}^{(0)}}-\boldsymbol{n}_{\mathrm{L}} \frac{\partial P_{\text {mech }}}{\partial n^{(0)}} \\
& +\boldsymbol{t}_{1} \cdot \frac{\partial \boldsymbol{\tau}}{\partial s_{1}^{(0)}}+\boldsymbol{n}_{\mathrm{L}} \cdot \frac{\partial \boldsymbol{\tau}}{\partial n^{(0)}}+\varepsilon \boldsymbol{t}_{1} \frac{\partial f_{0}}{\partial s_{1}^{(1)}}+\varepsilon \boldsymbol{n}_{\mathrm{L}} \frac{\partial f_{0}}{\partial n^{(1)}} \\
& -\varepsilon d\left(\boldsymbol{t}_{1} \frac{\partial \psi}{\partial s_{1}^{(1)}}+\boldsymbol{n}_{\mathrm{L}} \frac{\partial \psi}{\partial n^{(1)}}\right)\left(\kappa_{1} \frac{\partial \psi}{\partial n^{(1)}}+\frac{\partial^{2} \psi}{\partial s_{1}^{(1)^{2}}}+\frac{\partial^{2} \psi}{\partial n^{(1)^{2}}}\right)
\end{aligned}
$$

Here, we assume $\partial \tau / \partial s_{1}^{(0)}=0$ and $\partial P_{\text {mech }} / \partial s_{1}^{(0)}=0$ at the interface. Then, the integration of Eq. (15) from $-\infty$ to $+\infty$ along the $n$ axis gives

$$
\begin{aligned}
0 & =-\boldsymbol{n}_{\mathrm{L}} \int_{-\infty}^{+\infty} \frac{\partial P_{\text {mech }}}{\partial n} \mathrm{~d} n+\boldsymbol{n}_{\mathrm{L}} \cdot \int_{-\infty}^{+\infty} \frac{\partial \boldsymbol{\tau}}{\partial n} \mathrm{~d} n \\
& -\varepsilon \boldsymbol{t}_{1} \int_{-\infty}^{+\infty} \frac{\partial f_{0}}{\partial s_{1}} \mathrm{~d} n+\varepsilon \boldsymbol{n}_{\mathrm{L}} \int_{-\infty}^{+\infty} \frac{\partial f_{0}}{\partial n} \mathrm{~d} n \\
& -\boldsymbol{t}_{1} \varepsilon d \int_{-\infty}^{+\infty} \kappa_{1} \frac{\partial \psi}{\partial s_{1}} \frac{\partial \psi}{\partial n} \mathrm{~d} n-\boldsymbol{t}_{1} \frac{\varepsilon d}{2} \int_{-\infty}^{+\infty} \frac{\partial}{\partial s_{1}}\left(\frac{\partial \psi}{\partial s_{1}}\right)^{2} \mathrm{~d} n \\
& -\boldsymbol{t}_{1} \varepsilon d \int_{-\infty}^{+\infty} \frac{\partial}{\partial n}\left(\frac{\partial \psi}{\partial s_{1}} \frac{\partial \psi}{\partial n}\right) \mathrm{d} n+\boldsymbol{t}_{1} \frac{\varepsilon d}{2} \int_{-\infty}^{+\infty} \frac{\partial}{\partial s_{1}}\left(\frac{\partial \psi}{\partial n}\right)^{2} \mathrm{~d} n \\
& -\boldsymbol{n}_{\mathrm{L}} \varepsilon d \int_{-\infty}^{+\infty} \frac{\partial}{\partial s_{1}}\left(\frac{\partial \psi}{\partial n} \frac{\partial \psi}{\partial s_{1}}\right) \mathrm{d} n+\boldsymbol{n}_{\mathrm{L}} \frac{\varepsilon d}{2} \int_{-\infty}^{+\infty} \frac{\partial}{\partial n}\left(\frac{\partial \psi}{\partial s_{1}}\right)^{2} \mathrm{~d} n \\
& -\boldsymbol{n}_{\mathrm{L}} \varepsilon d \int_{-\infty}^{+\infty} \kappa_{1}\left(\frac{\partial \psi}{\partial n}\right)^{2} \mathrm{~d} n+\boldsymbol{n}_{\mathrm{L}} \frac{\varepsilon d}{2} \int_{-\infty}^{+\infty} \frac{\partial}{\partial n}\left(\frac{\partial \psi}{\partial n}\right)^{2} \mathrm{~d} n
\end{aligned}
$$


The superscripts in terms $n^{(0)}$ and $n^{(1)}$ in Eq. (15) can be omitted in Eq. (16) because there is no difference between the normal directions $n^{(0)}$ and $n^{(1)}$ over the integration. The first term on the right-hand side of Eq. (16) is calculated as follows:

$$
-\boldsymbol{n}_{\mathrm{L}} \int_{P_{\mathrm{G}}}^{P_{\mathrm{L}}} \mathrm{d} P_{\text {mech }}=-\boldsymbol{n}_{\mathrm{L}} P_{\mathrm{L}}-\boldsymbol{n}_{\mathrm{G}} P_{\mathrm{G}},
$$

and the second term is

$$
\boldsymbol{n}_{\mathrm{L}} \cdot \int_{\tau_{\mathrm{G}}}^{\tau_{\mathrm{L}}} \mathrm{d} \tau=\boldsymbol{n}_{\mathrm{L}} \cdot \tau_{\mathrm{L}}+\boldsymbol{n}_{\mathrm{G}} \cdot \tau_{\mathrm{G}}
$$

When $\partial \psi / \partial n=0$ at $n=-\infty$ or $+\infty$, we obtain

$$
\begin{aligned}
0 & =-\left[\left(-P_{\mathrm{G}}-\varepsilon \pi_{\mathrm{G}}\right) \boldsymbol{n}_{\mathrm{G}}+\boldsymbol{n}_{\mathrm{G}} \cdot \boldsymbol{\tau}_{\mathrm{G}}\right]-\left[\left(-P_{\mathrm{L}}-\varepsilon \pi_{\mathrm{L}}\right) \boldsymbol{n}_{\mathrm{L}}+\boldsymbol{n}_{\mathrm{L}} \cdot \boldsymbol{\tau}_{\mathrm{L}}\right] \\
& +\boldsymbol{n}_{\mathrm{L}} \varepsilon d \int_{-\infty}^{+\infty} \kappa_{1}\left(\frac{\partial \psi}{\partial n}\right)^{2} \mathrm{~d} n-\boldsymbol{t}_{1} \frac{\varepsilon d}{2} \int_{-\infty}^{+\infty} \frac{\partial}{\partial s_{1}}\left(\frac{\partial \psi}{\partial n}\right)^{2} \mathrm{~d} n \\
& +\boldsymbol{n}_{\mathrm{L}} \varepsilon d \int_{-\infty}^{+\infty} \frac{\partial}{\partial s_{1}}\left(\frac{\partial \psi}{\partial n} \frac{\partial \psi}{\partial s_{1}}\right) \mathrm{d} n-\boldsymbol{n}_{\mathrm{L}} \frac{\varepsilon d}{2} \int_{-\infty}^{+\infty} \frac{\partial}{\partial n}\left(\frac{\partial \psi}{\partial s_{1}}\right)^{2} \mathrm{~d} n \\
& -\boldsymbol{t}_{1} \int_{-\infty}^{+\infty} \varepsilon \frac{\partial \psi}{\partial s_{1}}\left(-a \psi+b \psi^{3}\right) \mathrm{d} n+\boldsymbol{t}_{1} \int_{-\infty}^{+\infty} \varepsilon \frac{\partial \pi_{\mathrm{i}}}{\partial s_{1}} \mathrm{~d} n \\
& +\boldsymbol{t}_{1} \frac{\varepsilon d}{2} \int_{-\infty}^{+\infty} \frac{\partial}{\partial s_{1}}\left(\frac{\partial \psi}{\partial s_{1}}\right)^{2} \mathrm{~d} n+\boldsymbol{t}_{1} \varepsilon d \int_{-\infty}^{+\infty} \kappa_{1} \frac{\partial \psi}{\partial s_{1}} \frac{\partial \psi}{\partial n} \mathrm{~d} n
\end{aligned}
$$

where $\pi_{i}=c z_{i} e V_{e} \psi\left[\mathrm{N} / \mathrm{m}^{2}\right]$. The function $\pi_{i}(i=\mathrm{L}$ or $\mathrm{G})$ represents contamination at the interface (liquid or gas phase). The surface tension coefficient under the flat surface is defined as follows (Rowlinson \& Widom, 1984):

$$
\sigma=d \int_{-\infty}^{\infty}\left(\frac{\partial \psi}{\partial n}\right)^{2} \mathrm{~d} n
$$

Equation (20) is substituted into Eq. (19) and is transformed in order to consider the relation between Eqs. (11) and (19).

Using Eq. (20), we can rewrite the third and fourth terms on the right-hand side of Eq. (19) as

$$
\begin{gathered}
\boldsymbol{n}_{\mathrm{L}} \varepsilon d \int_{-\infty}^{+\infty} \kappa_{1}\left(\frac{\partial \psi}{\partial n}\right)^{2} \mathrm{~d} n=\boldsymbol{n}_{\mathrm{L}} \varepsilon \sigma \kappa_{1} \\
\boldsymbol{t}_{1} \frac{\varepsilon d}{2} \int_{-\infty}^{+\infty} \frac{\partial}{\partial s_{1}}\left(\frac{\partial \psi}{\partial n}\right)^{2} \mathrm{~d} n \\
=\boldsymbol{t}_{1} \frac{\varepsilon}{2} \frac{\partial}{\partial s_{1}}\left[d \int_{-\infty}^{+\infty}\left(\frac{\partial \psi}{\partial n}\right)^{2} \mathrm{~d} n\right] \\
=\boldsymbol{t}_{1} \frac{\varepsilon}{2} \frac{\mathrm{d} \sigma}{\mathrm{d} s_{1}}
\end{gathered}
$$


In Eq. (21), although the curvature depends not only on the $s_{1}, s_{2}$ directions but also on the $n$ direction, as shown in Fig. 5, we do not consider the change in the curvature in the normal direction because the interface is very thin. In Eq. (22), the replacement of $\partial / \partial s_{1}=0$ by $\mathrm{d} / \mathrm{d} s_{1}=0$ means that the dependency of the surface tension coefficient on the $s_{2}$ coordinate is not considered. After substituting Eqs. (20), (21), and (22) into Eq. (19) and adding the terms with respect to the $s_{2}$ coordinate in Eq. (19), we derive the following equation:

$$
\begin{aligned}
& 0=\underbrace{-\left[\left(-P_{\mathrm{G}}-\varepsilon \pi_{\mathrm{G}}\right) \boldsymbol{n}_{\mathrm{G}}+\boldsymbol{n}_{\mathrm{G}} \cdot \boldsymbol{\tau}_{\mathrm{G}}\right]}_{(a)} \underbrace{-\left[\left(-P_{\mathrm{L}}-\varepsilon \pi_{\mathrm{L}}\right) \boldsymbol{n}_{\mathrm{L}}+\boldsymbol{n}_{\mathrm{L}} \cdot \boldsymbol{\tau}_{\mathrm{L}}\right]}_{(b)} \underbrace{-\boldsymbol{n}_{\mathrm{G}} \varepsilon\left(\kappa_{1}+\kappa_{2}\right) \sigma}_{(c)} \\
& \underbrace{-\varepsilon\left(\boldsymbol{t}_{1} \frac{1}{2} \frac{\partial \sigma}{\partial s_{1}}+\boldsymbol{t}_{2} \frac{1}{2} \frac{\partial \sigma}{\partial s_{2}}\right)}_{(d)} \underbrace{-\boldsymbol{n}_{\mathrm{G}} \varepsilon d \int_{-\infty}^{+\infty}\left[\frac{\partial}{\partial s_{1}}\left(\frac{\partial \psi}{\partial n} \frac{\partial \psi}{\partial s_{1}}\right)+\frac{\partial}{\partial s_{2}}\left(\frac{\partial \psi}{\partial n} \frac{\partial \psi}{\partial s_{2}}\right)\right] \mathrm{d} n}_{(e)} \\
& \underbrace{+\boldsymbol{n}_{\mathrm{G}} \varepsilon d \int_{-\infty}^{+\infty} \frac{1}{2} \frac{\partial}{\partial n}\left[\left(\frac{\partial \psi}{\partial s_{1}}\right)^{2}+\left(\frac{\partial \psi}{\partial s_{2}}\right)^{2}\right] \mathrm{d} n}_{(f)} \underbrace{-\varepsilon \int_{-\infty}^{+\infty}\left(\boldsymbol{t}_{1} \frac{\partial \psi}{\partial s_{1}}+\boldsymbol{t}_{2} \frac{\partial \psi}{\partial s_{2}}\right)\left(-a \psi+b \psi^{3}\right) \mathrm{d} n}_{(g)} \\
& \underbrace{+\varepsilon \int_{-\infty}^{+\infty}\left(\boldsymbol{t}_{1} \frac{\partial \pi_{\mathrm{i}}}{\partial s_{1}}+\boldsymbol{t}_{2} \frac{\partial \pi_{\mathrm{i}}}{\partial s_{2}}\right) \mathrm{d} n}_{(h)} \underbrace{+\frac{\varepsilon d}{2} \int_{-\infty}^{+\infty}\left[\boldsymbol{t}_{1} \frac{\partial}{\partial s_{1}}\left(\frac{\partial \psi}{\partial s_{1}}\right)^{2}+\boldsymbol{t}_{2} \frac{\partial}{\partial s_{2}}\left(\frac{\partial \psi}{\partial s_{2}}\right)^{2}\right] \mathrm{d} n}_{(i)} \\
& \underbrace{+\varepsilon d\left(\kappa_{1}+\kappa_{2}\right) \int_{-\infty}^{+\infty}\left(\boldsymbol{t}_{1} \frac{\partial \psi}{\partial s_{1}}+\boldsymbol{t}_{2} \frac{\partial \psi}{\partial s_{2}}\right)\left(\frac{\partial \psi}{\partial n}\right) \mathrm{d} n}_{(j)} \\
& \underbrace{+\varepsilon d \int_{-\infty}^{+\infty}\left[\boldsymbol{t}_{1} \frac{\partial}{\partial s_{2}}\left(\frac{\partial \psi}{\partial s_{1}} \frac{\partial \psi}{\partial s_{2}}\right)+\boldsymbol{t}_{2} \frac{\partial}{\partial s_{1}}\left(\frac{\partial \psi}{\partial s_{1}} \frac{\partial \psi}{\partial s_{2}}\right)\right] \mathrm{d} n}_{(k)} \\
& \underbrace{-\frac{\varepsilon d}{2} \int_{-\infty}^{+\infty}\left[\boldsymbol{t}_{1} \frac{\partial}{\partial s_{1}}\left(\frac{\partial \psi}{\partial s_{2}}\right)^{2}+\boldsymbol{t}_{2} \frac{\partial}{\partial s_{2}}\left(\frac{\partial \psi}{\partial s_{1}}\right)^{2}\right] \mathrm{d} n}_{(l)}
\end{aligned}
$$

This equation is the thermodynamic jump condition at the interface and is obtained by assuming that many elemental interfaces lie on the interface with a finite thickness and integrating it over the normal direction. The free energy is defined at the interface and is derived from the microscopic viewpoint (Hamiltonian) (Yonemoto \& Kunugi, 2010a). Therefore, Equation (23) considers multiple scales from microscopic to macroscopic. In order of appearance, the physical meaning of each term in Eq. (23) is discussed as follows:

a. The effects of the pressure, contamination at the interface, and shear stress caused by the fluid motion (gas phase side) on the interface

b. The effects of the pressure, contamination at the interface, and shear stress caused by the fluid motion (liquid phase side) on the interface

c. The conventional surface tension, known as the Young-Laplace formula

d. The gradient of the surface tension in the tangential direction; this term differs from the conventional term in Eq. (11), and we discuss this difference in Section 3.4 
e. The synergy effect of the change in $\psi$ in the normal and tangential directions at the interface

f. The change in the normal direction with respect to the $\psi$ gradient in the tangential direction; this term arises if the $\psi$ gradient changes in the tangential direction at the interface of the gas or liquid phase

g. The Marangoni effect term, which depends on the temperature difference because of the temperature-dependent coefficients $a$ and $b$

h. The effect of contamination in the tangential direction

i. The change in the tangential direction with respect to the $\psi$ gradient in the tangential direction; this term may simply be the tensile force

j. The synergy effect, which is the same as term (e); however, this term is slightly different from term (e) because this term includes the curvature

k. The synergy effect of the change in the tangential direction with respect to the $\psi$ gradient in the $s_{1}$ and $s_{2}$ tangential directions

1. The effect of the change in the tangential direction with respect to the $\psi$ gradient in the tangential direction; this term may simply be the tensile force, and the tangential directions are different from term (i).

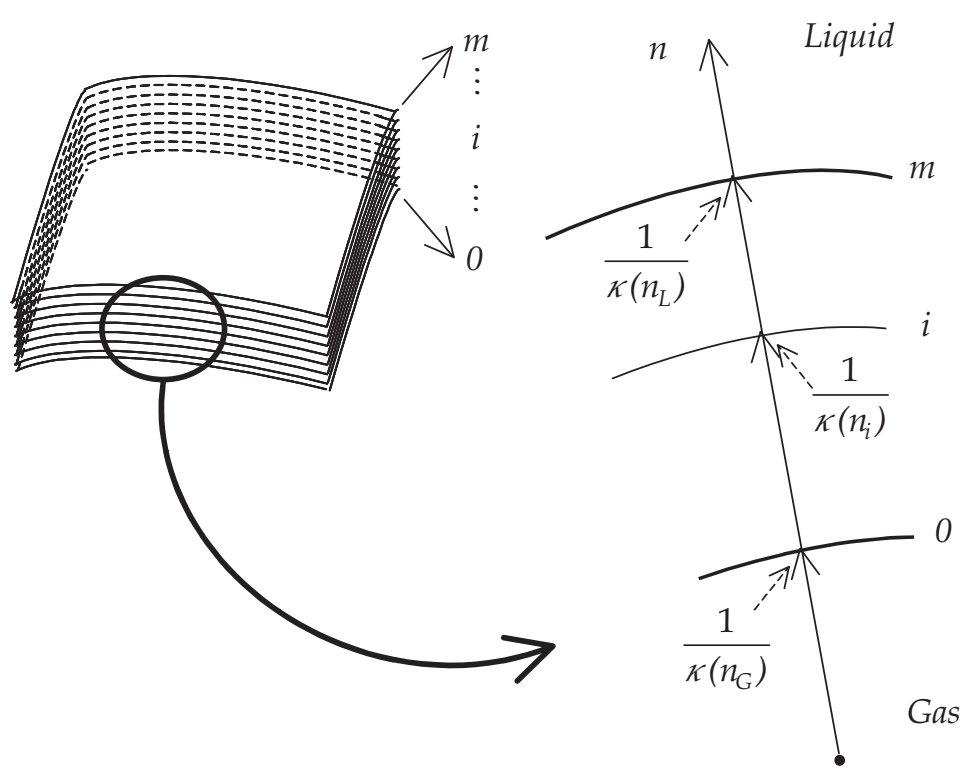

Fig. 5. Curvature through the interface

\subsection{Order estimation of jump condition}

We now consider the order estimation of the thermodynamic jump condition. The characteristic velocity, length, density, viscosity, interfacial thickness, surface tension coefficient, and electrostatic potential at the interface are $U_{0}[\mathrm{~m} / \mathrm{s}], L[\mathrm{~m}], \bar{\rho}=\left(\rho_{l}+\rho_{g}\right) / 2$ $\left[\mathrm{kg} / \mathrm{m}^{3}\right], \mu_{0}[\mathrm{~kg} / \mathrm{ms}], \delta[\mathrm{m}], \sigma_{0}[\mathrm{~N} / \mathrm{m}]$, and $V_{0}[\mathrm{~V}]$, respectively. The characteristic pressure is $\bar{\rho} U_{0}^{2}$ or $P_{0}\left[\mathrm{~N} / \mathrm{m}^{2}\right]$, and the interfacial wavelength is $\lambda_{1}$ (s $s_{1}$ axis) or $\lambda_{2}$ (s $s_{2}$ axis) [m]. 
First, Equation (23) is divided by $\bar{\rho} U_{0}^{2}$ and then normalized by other characteristic values. In particular, we normalize $n, \kappa_{1}$, and $\kappa_{2}$ by $\delta$ and $s_{1}$ and $s_{2}$ by $\lambda_{1}$ and $\lambda_{2}$, respectively. This normalization reduces Eq. (23) to

$$
\begin{aligned}
& 0=\underbrace{-\left[\left(-\frac{1}{\mathrm{E}} P_{\mathrm{G}}^{*}-\frac{1}{4 \mathrm{~N}} \pi_{\mathrm{G}}^{*}\right) \boldsymbol{n}_{\mathrm{G}}+\frac{1}{\mathrm{Re}} \boldsymbol{n}_{\mathrm{G}} \cdot \tau_{\mathrm{G}}^{*}\right]}_{(a)^{\prime}} \underbrace{\left[\left(-\frac{1}{\mathrm{E}} P_{\mathrm{L}}^{*}-\frac{1}{4 \mathrm{~N}} \pi_{\mathrm{L}}^{*}\right) \boldsymbol{n}_{\mathrm{L}}+\frac{1}{\mathrm{Re}} \boldsymbol{n}_{\mathrm{L}} \cdot \tau_{\mathrm{L}}^{*}\right]}_{(b)^{\prime}} \\
& \underbrace{-\boldsymbol{n}_{\mathrm{G}} \frac{1}{\mathrm{We}}\left(\kappa_{1}^{*}+\kappa_{2}^{*}\right) \sigma^{*}}_{(c)^{\prime}}-\underbrace{-\varepsilon\left(\boldsymbol{t}_{1} \frac{1}{\mathrm{We}_{1}} \frac{1}{2} \frac{\partial \sigma^{*}}{\partial s_{1}^{*}}+\boldsymbol{t}_{2} \frac{1}{\mathrm{We}_{2}} \frac{1}{2} \frac{\partial \sigma^{*}}{\partial s_{2}^{*}}\right)}_{(d)^{\prime}} \\
& \underbrace{-\boldsymbol{n}_{\mathrm{G}} \varepsilon d^{*} \int_{-\infty}^{+\infty}\left[\frac{\mathrm{Kn}_{1}}{\mathrm{We}_{1}} \frac{\partial}{\partial s_{1}^{*}}\left(\frac{\partial \psi}{\partial n^{*}} \frac{\partial \psi}{\partial s_{1}^{*}}\right)+\frac{\mathrm{Kn}_{2}}{\mathrm{We}_{2}} \frac{\partial}{\partial s_{2}^{*}}\left(\frac{\partial \psi}{\partial n^{*}} \frac{\partial \psi}{\partial s_{2}^{*}}\right)\right] \mathrm{d} n^{*}}_{(e)^{\prime}} \\
& \underbrace{+\boldsymbol{n}_{\mathrm{G}} \varepsilon d^{*} \int_{-\infty}^{+\infty} \frac{1}{2} \frac{\partial}{\partial n^{*}}\left[\frac{\mathrm{Kn}_{1}}{\mathrm{We}_{1}}\left(\frac{\partial \psi}{\partial s_{1}^{*}}\right)^{2}+\frac{\mathrm{Kn}_{2}}{\mathrm{We}_{2}}\left(\frac{\partial \psi}{\partial s_{2}^{*}}\right)^{2}\right] \mathrm{d} n^{*}}_{(f)^{\prime}} \\
& \underbrace{-\varepsilon \int_{-\infty}^{+\infty}\left(\boldsymbol{t}_{1} \frac{\mathrm{Kn}_{1}}{\mathrm{E}} \frac{\partial \psi}{\partial s_{1}^{*}}+\boldsymbol{t}_{2} \frac{\mathrm{Kn}_{2}}{\mathrm{E}} \frac{\partial \psi}{\partial s_{2}^{*}}\right)\left(-a^{*} \psi+b^{*} \psi^{3}\right) \mathrm{d} n^{*}}_{(g)^{\prime}} \\
& \underbrace{+\int_{-\infty}^{+\infty}\left(\boldsymbol{t}_{1} \frac{\mathrm{Kn}}{4 \mathrm{~N}} \frac{\partial \pi_{\mathrm{i}}^{*}}{\partial s_{1}^{*}}+\boldsymbol{t}_{2} \frac{\mathrm{Kn}_{2}}{4 \mathrm{~N}} \frac{\partial \pi_{\mathrm{i}}^{*}}{\partial s_{2}^{*}}\right) \mathrm{d} n^{*}}_{(h)^{\prime}} \\
& \underbrace{+\frac{\varepsilon d^{*}}{2} \int_{-\infty}^{+\infty}\left[\boldsymbol{t}_{1} \frac{\mathrm{Kn}_{1}}{\mathrm{We}_{1}} \frac{\partial}{\partial s_{1}^{*}}\left(\frac{\partial \psi}{\partial s_{1}^{*}}\right)^{2}+\boldsymbol{t}_{2} \frac{\mathrm{Kn}_{2}}{\mathrm{We}_{2}} \frac{\partial}{\partial s_{2}^{*}}\left(\frac{\partial \psi}{\partial s_{2}^{*}}\right)^{2}\right] \mathrm{d} n^{*}}_{(i)^{\prime}}
\end{aligned}
$$

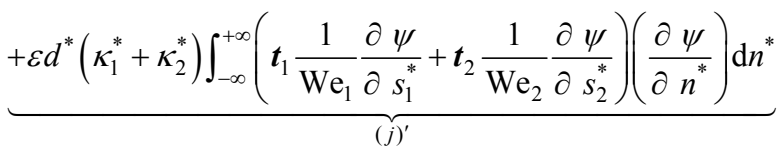

$$
\begin{aligned}
& \underbrace{+\varepsilon d^{*} \int_{-\infty}^{+\infty}\left[\boldsymbol{t}_{1} \frac{\mathrm{Kn}_{2}^{2}}{\mathrm{We}_{1}} \frac{\partial}{\partial s_{2}^{*}}\left(\frac{\partial \psi}{\partial s_{1}^{*}} \frac{\partial \psi}{\partial s_{2}^{*}}\right)+\boldsymbol{t}_{2} \frac{\mathrm{Kn}_{1}^{2}}{\mathrm{We}_{2}} \frac{\partial}{\partial s_{1}^{*}}\left(\frac{\partial \psi}{\partial s_{1}^{*}} \frac{\partial \psi}{\partial s_{2}^{*}}\right)\right] \mathrm{d} n^{*}}_{(k)^{\prime}} \\
& \underbrace{-\frac{\varepsilon d^{*}}{2} \int_{-\infty}^{+\infty}\left[\boldsymbol{t}_{1} \frac{\mathrm{Kn}_{2}^{2}}{\mathrm{We}_{1}} \frac{\partial}{\partial s_{1}^{*}}\left(\frac{\partial \psi}{\partial s_{2}^{*}}\right)^{2}+\boldsymbol{t}_{2} \frac{\mathrm{Kn}_{1}^{2}}{\mathrm{We}_{2}} \frac{\partial}{\partial s_{2}^{*}}\left(\frac{\partial \psi}{\partial s_{1}^{*}}\right)^{2}\right] \mathrm{d} n^{*} .}_{(l)^{\prime}}
\end{aligned}
$$

In Eq. (24), the dimensionless numbers are 


$$
\begin{array}{ll}
\mathrm{E}=\frac{\bar{\rho} U_{0}^{2}}{P_{0}} \quad \mathrm{~N}=\frac{L \delta^{2} \bar{\rho} U_{0}^{2}}{z_{\mathrm{i}} e V_{0}} \quad \mathrm{Re}=\frac{\bar{\rho} U_{0} L}{\mu_{0}} \quad \mathrm{We}=\frac{\bar{\rho} U_{0}^{2} \delta}{\sigma_{0}} \quad(25 \mathrm{a}, \mathrm{b}, \mathrm{c}, \mathrm{d}) \\
\mathrm{We}_{1}=\frac{\bar{\rho} U_{0}^{2} \lambda_{1}}{\sigma_{0}} \quad \mathrm{We}_{2}=\frac{\bar{\rho} U_{0}^{2} \lambda_{2}}{\sigma_{0}} \quad \mathrm{Kn}_{1}=\frac{\delta}{\lambda_{1}} \quad \mathrm{Kn}_{2}=\frac{\delta}{\lambda_{2}} \quad(26 \mathrm{a}, \mathrm{b}, \mathrm{c}, \mathrm{d})
\end{array}
$$

Of these equations, we especially focus on Eq. (25b).

Focusing on the electrical repulsive and hydrodynamic forces, we discuss a situation in which two bubbles either coalesce or bounce off each other. In terms $(a)^{\prime},(b)$ ', and $(h)^{\prime}$ of Eq. (24), the dimensionless number $\mathrm{N}$ represents the relationship between the electrostatic force due to contamination at the interface and the hydrodynamic force. With respect to the dimensionless number $\mathrm{N}$, we assume a situation in which bubbles $\mathrm{A}$ and $\mathrm{B}$ interact and there is a difference in the velocities of the bubbles, as shown in Fig. 6. Here, we simply take the interfacial electrostatic potential of an experimental value as the measured $\zeta$ potential of gas bubbles (Graciaa et al., 1995). The measured $\zeta$ potential is on the order of tens of millivolts. The density is $10^{3}\left[\mathrm{~kg} / \mathrm{m}^{3}\right]$, and $\delta$ is $10^{-9}[\mathrm{~m}]$. The elementary charge is $10^{-19}[\mathrm{C}]$. If the characteristic length $L$ corresponding to the bubble diameter is 1 [mm], and the characteristic velocity corresponding to the relative bubble velocity between bubbles $\mathrm{A}$ and B is $1[\mathrm{~m} / \mathrm{s}]$, then the order of the inverse of the dimensionless number $\mathrm{N}$ in terms $(a)^{\prime},(b)^{\prime}$, and $(h)^{\prime}$ becomes $10^{-2}$. This means that the electrostatic force due to contamination can be ignored when compared with the hydrodynamic force. However, if the characteristic velocity is $0.01[\mathrm{~m} / \mathrm{s}]$, the order of the inverse of the dimensionless number $\mathrm{N}$ becomes $10^{2}$, and the electrostatic force due to contamination is significant when compared to the hydrodynamic force. This order estimation indicates that the dimensionless number $\mathrm{N}$ may be important for evaluating bubble coalescence and repulsion when focusing on both the electrostatic potential at the interface and the bubble velocity. In contrast, assume a situation where the relative bubble velocity between bubbles A and B is 0, as shown in Fig. 6 (i.e., the

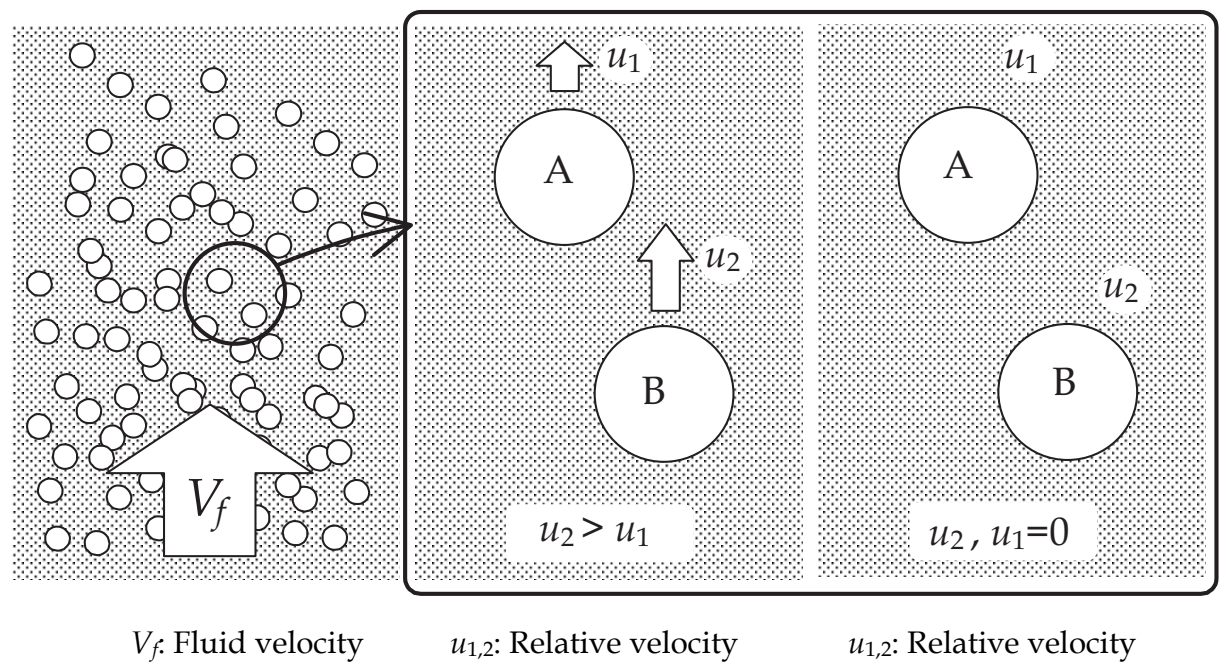

Fig. 6. Schematic of two microbubbles 
characteristic velocity is the bubble velocity). If the bubble velocity approaches zero and the bubble diameter decreases, the inverse of the dimensionless number $\mathrm{N}$ increases. This indicates that the electrostatic force due to contamination at the interface is significant when compared to the hydrodynamic force, although it depends on the magnitude of the electrostatic potential at the interface. In other words, it is difficult to deform the bubble; it maintains a spherical shape except for the effect of the electrostatic force or mass transfer at the interface. In this situation, the dimensionless number $\mathrm{N}$ roughly explains that the breakup of a small bubble has difficult occurring if the bubble diameter is very small.

\subsection{Consideration of surface tension gradient}

The thermodynamic jump condition seems to be the same as the conventional jump condition when we focus on terms (a)-(d) in Eq. (23). However, the detailed formula of (d) differs from that of the conventional one. In this section, we discuss term (d).

The interfacial coordinate system shown in Fig. 7 is reconsidered using the same notations as in Section 3.2: $n, s_{1}$, and $s_{2}$. Vector $\boldsymbol{t}$ is defined on the $l$ axis and consists of $\boldsymbol{t}_{1}$ and $\boldsymbol{t}_{2}$. Unit vectors $\boldsymbol{t}_{1}$ and $\boldsymbol{t}_{2}$ are the tangential vectors of the $s_{1}$ and $s_{2}$ axes, respectively. Here, $\boldsymbol{t}=\boldsymbol{t}_{1}+\boldsymbol{t}_{2}$. However, the coordinate system in Fig. 7 has different implications from the implication in Fig. 4: the interface shown in Fig. 7 is a statistical interface obtained after integrating the elemental interfaces of Fig. 4. This means that the integrated interface is the macroscopic interface in which the interface can be recognized geometrically, such as a plane or spherical surface. Based on these concepts, we discuss term (d).

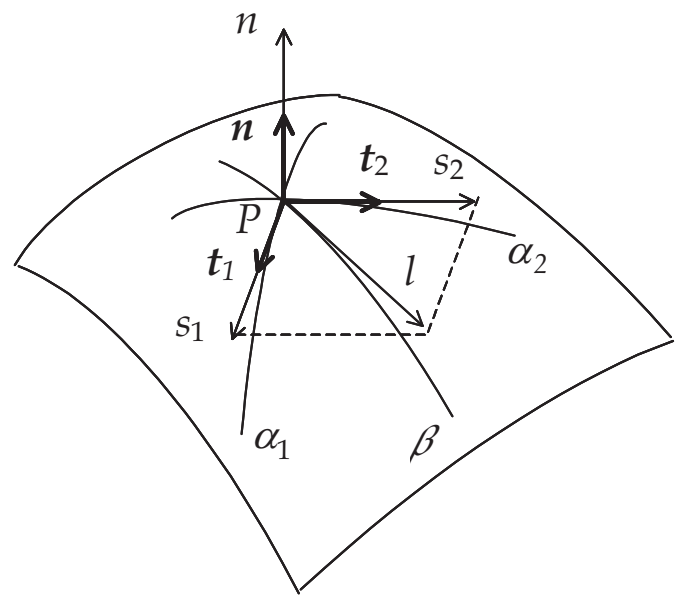

Fig. 7. Statistical local interface

Let $\boldsymbol{f}$ denote term (d) in Eq. (23). This term can be expressed as

$$
f=f_{1}+f_{2}
$$

Equation (27) is a resultant force with respect to the gradient of surface tension in the tangential direction. In this equation, force $f_{1}$ is the gradient of the surface tension defined on the $s_{1}$ axis, and force $\boldsymbol{f}_{2}$ is defined on the $s_{2}$ axis as follows: 


$$
\begin{aligned}
& f_{1}=\boldsymbol{t}_{1} \frac{1}{2} \frac{\partial \sigma}{\partial s_{1}} \\
& \boldsymbol{f}_{2}=\boldsymbol{t}_{2} \frac{1}{2} \frac{\partial \sigma}{\partial s_{2}}
\end{aligned}
$$
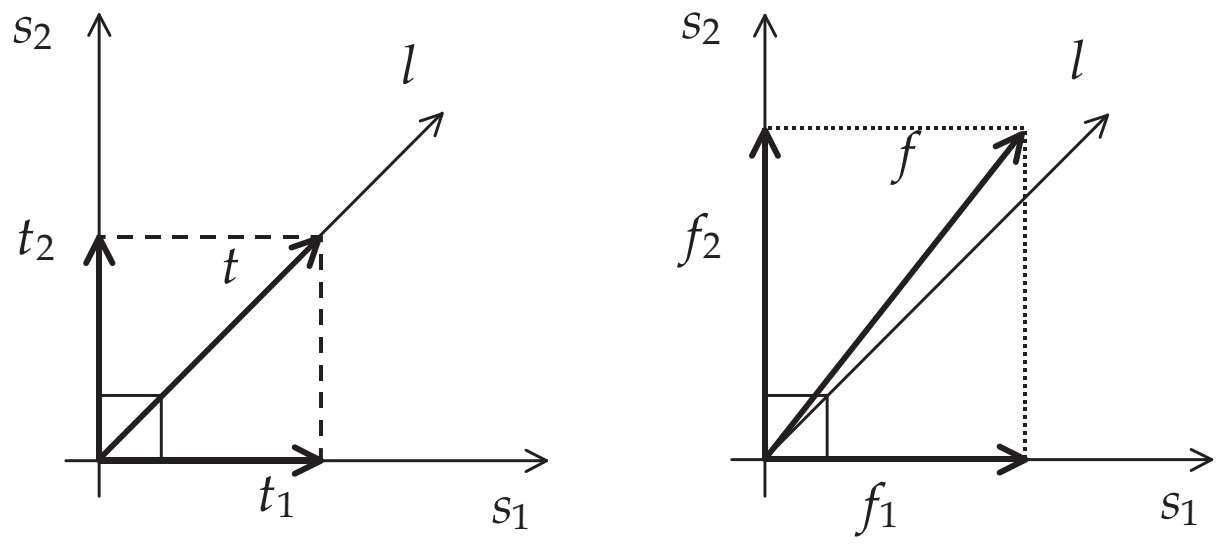

Fig. 8. Vector diagram in the $\mathrm{s}_{1}-\mathrm{s}_{2}$ tangential plane: (a) Unit base vector $t_{1}, t_{2}\left(t=t_{1}+t_{2}\right)$, (b) Surface force $f_{1}, f_{2}\left(f=f_{1}+f_{2}\right)$

Therefore, an image of Eq. (27) is represented by a vector diagram, as shown in Fig. 8. The direction of $f$ is arbitrary in the $s_{1}-s_{2}$ plane, as shown in Fig. 8b, because the magnitude of $f_{1}$ is not always equal to that of $f_{2}$. However, if the magnitude of $f_{1}$ is equal to that of $\boldsymbol{f}_{2}$, then

$$
\frac{\partial \sigma}{\partial s_{1}}=\frac{\partial \sigma}{\partial s_{2}}
$$

This equation indicates that the direction of $f$ is parallel to that of the $l$ axis. Therefore, the resultant force $\boldsymbol{f}$ is revaluated by using vector $\tilde{\boldsymbol{t}}$, which is a unit tangential vector of the $l$ axis. Eventually, Equation (27) is transformed as follows:

$$
\begin{aligned}
\boldsymbol{f} & =\left(\boldsymbol{t}_{1} \frac{1}{2} \frac{\partial \sigma}{\partial s_{1}}+\boldsymbol{t}_{2} \frac{1}{2} \frac{\partial \sigma}{\partial s_{2}}\right) \\
& =\frac{1}{2} \frac{\mathrm{d} \sigma}{\mathrm{d} l}\left(\boldsymbol{t}_{1} \frac{\partial l}{\partial s_{1}}+\boldsymbol{t}_{2} \frac{\partial l}{\partial s_{2}}\right) \\
& =\frac{\sqrt{2}}{2} \frac{\mathrm{d} \sigma}{\mathrm{d} l}\left(\boldsymbol{t}_{1}+\boldsymbol{t}_{2}\right) \\
& =\frac{\mathrm{d} \sigma}{\mathrm{d} l} \tilde{\boldsymbol{t}}
\end{aligned}
$$


In this equation, $\tilde{\boldsymbol{t}}$ is $\boldsymbol{t} /|\boldsymbol{t}|$. By replacing term (d) in Eq. (23) with Eq. (31), we establish that the thermodynamic jump condition agrees with the conventional condition except for terms (e)-(j) in Eq. (23). This result suggests that the conventional jump condition holds under the conditions of Eqs. (20) and (30). Therefore, the conventional jump condition is restricted to spherical bubbles or droplets and is inaccurate when the relationship between the surface tensions of the $s_{1}$ and $s_{2}$ directions is imbalanced.

\section{Consideration of Kelvin equation}

We consider the equilibrium state where the gas and liquid phases coexist and temperature is constant. The gas-liquid interface is a flat surface. In this situation, the amount of evaporated liquid from the gas phase to the liquid phase is determined by the relationship between the saturated vapor pressure and ambient pressure if the temperature is constant. The vapor in the gas phase condenses to the liquid phase when the ambient pressure is increased to greater than the saturated vapor pressure. On the other hand, the liquid evaporates to the gas phase when the ambient pressure is decreased to less than the saturated vapor pressure. This is formulated by considering the changes in chemical potential thermodynamically. The same discussion is applied to a bubble and droplet. However, a bubble or droplet has a curvature. The vapor pressure of a droplet takes a different value when the interface has a curvature. The concrete equation is as follows (Butt et al., 2003).

$$
\ln \left(\frac{P_{\kappa}}{P^{*}}\right)=\frac{2 H \sigma V_{m}}{\mathrm{R} T}
$$

In this equation, $P^{*}$ and $P_{\kappa}$ represent the vapor pressures where the gas-liquid interface is flat and with a curvature, respectively. $H\left[1 / \mathrm{m}^{3}\right], \sigma[\mathrm{N} / \mathrm{m}], V_{m}\left[\mathrm{~m}^{3} / \mathrm{kg}\right], \mathrm{R}[\mathrm{J} / \mathrm{kg} \mathrm{K}]$, and $T[\mathrm{~K}]$ are the mean curvature, surface tension coefficient, specific volume, gas constant, and temperature, respectively. Equation (32) is mainly derived based on both the Gibbs-Duhem equation and Young-Laplace equation from the thermodynamic point of view. In this section, we show that the Kelvin equation can be derived from the multi-scale multiphase flow equation.

\subsection{Derivation of Kelvin equation from multi-scale multiphase flow equation}

The Chapman-Enskog expansion is applied to the conventional Navier-Stokes equation to derive the multi-scale multiphase flow equation from which the thermodynamic interfacial jump condition is finally derived. In this section, the Kelvin equation is derived using the same procedure.

Figure 9 shows a schematic of a multi-scale concept around the interface. $O(1)$ represents the order of a macroscopic scale. $\varepsilon$ and $\varepsilon^{2}$ represent mesoscopic scales: the scale of $\varepsilon$ is smaller than that of $\varepsilon^{2}$. However, we assume that the continuum approximation holds in each scale. Based on this assumption, we consider the Kelvin equation.

By considering the Chapman-Enskog expansion in Eq. (7) and $\varepsilon$ until the second order, the following equation is derived.

$$
\rho \frac{\mathrm{D} \boldsymbol{u}}{\mathrm{D} t^{(0)}}+\varepsilon \rho \frac{\mathrm{D} \boldsymbol{u}^{\prime}}{\mathrm{D} t^{(1)}}+\varepsilon^{2} \rho \frac{\mathrm{D} \boldsymbol{u}^{\prime \prime}}{\mathrm{D} t^{(2)}}=-\nabla^{(0)} \cdot \boldsymbol{T}-\varepsilon \nabla^{(1)} \cdot \boldsymbol{T}^{\prime}-\varepsilon^{2} \nabla^{(2)} \cdot \boldsymbol{T}^{\prime \prime}+\rho \boldsymbol{g}
$$


Here, the diffusion process is proportional to the gradient of the chemical potential (Onsager, 1931a, 1931b), which yields the thermodynamic driving force as follows:

$$
\begin{aligned}
\boldsymbol{F}^{\prime \prime} & =-\rho \boldsymbol{\nabla} \frac{\delta F}{\delta \tilde{\eta}} \\
& =-\rho \nabla \mu
\end{aligned}
$$

The diffusion flux $(J)$ is then represented by

$$
\boldsymbol{J}=-m C \nabla \mu
$$

where, $m$ and $C$ are the mobility $[\mathrm{m} \cdot \mathrm{mol} / \mathrm{N} \cdot \mathrm{s}]$ and $\mathrm{mol}$ concentration $\left[\mathrm{mol} / \mathrm{m}^{3}\right]$, respectively. In the present study, we assumed that the thermodynamic force (Eq. (34)) corresponds to $T^{\prime \prime}$ in Eq. (33) as follows:

$$
-\varepsilon^{2} \nabla^{(2)} \cdot \boldsymbol{T}^{\prime \prime}=\varepsilon^{2} \boldsymbol{F}^{\prime \prime}
$$

In Eq. (33), the order of $\varepsilon$ is smaller than that of $O(1)$. Thus, the order of $\varepsilon^{2}$ is smaller than that of $\varepsilon$. Therefore, an interfacial phenomena characterized by $\varepsilon^{2}$ can be negligible compared to that of $O(1)$.

However, the driving force (Eq. (34)) characterized by small scales such as $\varepsilon^{2}$ will affect phenomena at large scales such as $O(1)$ after enough time, even if there are differences between the scales in Eq. (33).

Considering Eqs. (33), (34), and (36) and performing a simple tensor analysis, such as the derivation of Eq. (10), gives

$$
\begin{aligned}
\rho \frac{\mathrm{D} \boldsymbol{u}}{\mathrm{D} t^{(0)}}+\varepsilon \rho \frac{\mathrm{D} \boldsymbol{u}^{\prime}}{\mathrm{D} t^{(1)}}+\varepsilon^{2} \rho \frac{\mathrm{D} \boldsymbol{u}^{\prime \prime}}{\mathrm{D} t^{(2)}} & =-\nabla^{(0)} \cdot \boldsymbol{T}+\varepsilon \boldsymbol{\nabla}^{(1)} \cdot\left(f_{0}(\psi) \boldsymbol{I}\right) \\
& -\varepsilon d \boldsymbol{\nabla}^{(1)} \psi\left(\boldsymbol{\nabla}^{(1)} \cdot \boldsymbol{\nabla}^{(1)} \psi\right)-\varepsilon^{2} \rho \boldsymbol{\nabla}^{(2)} \mu+\rho \boldsymbol{g}
\end{aligned}
$$

Equation (37) is transformed into the interfacial coordinates system in a similar manner to that discussed in section 3.2. For the sake of simplicity, we show the transformation of the fourth term on the right-hand side of Eq. (37). Referring to Eq. (13) in section 3.2, the fourth term on the right-hand side of Eq. (37) is transformed as follows:

$$
-\varepsilon^{2} \rho \nabla^{(2)} \mu \rightarrow-\varepsilon^{2} \rho\left(\boldsymbol{t}_{1} \frac{\partial \mu}{\partial s_{1}^{(2)}}+\boldsymbol{t}_{2} \frac{\partial \mu}{\partial s_{2}^{(2)}}+\boldsymbol{n}_{\mathrm{L}} \frac{\partial \mu}{\partial n^{(2)}}\right)
$$

Integration of this equation over the interface gives

$$
-\varepsilon^{2} \rho \int_{-\infty}^{\infty}\left(\boldsymbol{t}_{1} \frac{\partial \mu}{\partial s_{1}^{(2)}}+\boldsymbol{t}_{2} \frac{\partial \mu}{\partial s_{2}^{(2)}}\right) \mathrm{d} n-\varepsilon^{2} \rho \boldsymbol{n}_{\mathrm{L}} \int_{-\infty}^{\infty} \frac{\partial \mu}{\partial n^{(2)}} \mathrm{d} n
$$

In this calculation, chemical potential is assumed to be constant along the interface in tangential direction. Therefore, the first term in Eq. (39) is omitted. Thus, Equation (38) is calculated as follows: 


$$
-\varepsilon^{2} \rho \boldsymbol{\nabla}^{(2)} \mu=-\varepsilon^{2} \rho \boldsymbol{n}_{\mathrm{L}} \int_{-\infty}^{\infty} \frac{\partial \mu}{\partial n^{(2)}} \mathrm{d} n
$$

Finally, Equation (37) is transformed into the interfacial coordinates system as follows:

$$
\begin{aligned}
& 0=\underbrace{-\left[\left(-P_{\mathrm{G}}-\varepsilon \pi_{\mathrm{G}}\right) \boldsymbol{n}_{\mathrm{G}}+\boldsymbol{n}_{\mathrm{G}} \cdot \boldsymbol{\tau}_{\mathrm{G}}\right]}_{(a)} \underbrace{-\left[\left(-P_{\mathrm{L}}-\varepsilon \pi_{\mathrm{L}}\right) \boldsymbol{n}_{\mathrm{L}}+\boldsymbol{n}_{\mathrm{L}} \cdot \boldsymbol{\tau}_{\mathrm{L}}\right]}_{(b)} \underbrace{-\boldsymbol{n}_{\mathrm{G}} \varepsilon\left(\kappa_{1}+\kappa_{2}\right) \sigma}_{(c)} \\
& \underbrace{-\varepsilon\left(\boldsymbol{t}_{1} \frac{1}{2} \frac{\partial \sigma}{\partial s_{1}^{(1)}}+\boldsymbol{t}_{2} \frac{1}{2} \frac{\partial \sigma}{\partial s_{2}^{(1)}}\right)}_{(d)} \underbrace{-\boldsymbol{n}_{\mathrm{G}} \varepsilon d \int_{-\infty}^{+\infty}\left[\frac{\partial}{\partial s_{1}^{(1)}}\left(\frac{\partial \psi}{\partial n^{(1)}} \frac{\partial \psi}{\partial s_{1}^{(1)}}\right)+\frac{\partial}{\partial s_{2}^{(1)}}\left(\frac{\partial \psi}{\partial n^{(1)}} \frac{\partial \psi}{\partial s_{2}^{(1)}}\right)\right] \mathrm{d} n}_{(e)} \\
& \underbrace{+\boldsymbol{n}_{\mathrm{G}} \varepsilon d \int_{-\infty}^{+\infty} \frac{1}{2} \frac{\partial}{\partial n^{(1)}}\left[\left(\frac{\partial \psi}{\partial s_{1}^{(1)}}\right)^{2}+\left(\frac{\partial \psi}{\partial s_{2}^{(1)}}\right)^{2}\right]}_{(f)} \mathrm{d} n \underbrace{-\varepsilon \int_{-\infty}^{+\infty}\left(\boldsymbol{t}_{1} \frac{\partial \psi}{\partial s_{1}^{(1)}}+\boldsymbol{t}_{2} \frac{\partial \psi}{\partial s_{2}^{(1)}}\right)\left(-a \psi+b \psi^{3}\right) \mathrm{d} n}_{(g)} \\
& \underbrace{+\varepsilon \int_{-\infty}^{+\infty}\left(\boldsymbol{t}_{1} \frac{\partial \pi_{\mathrm{i}}}{\partial s_{1}^{(1)}}+\boldsymbol{t}_{2} \frac{\partial \pi_{\mathrm{i}}}{\partial s_{2}^{(1)}}\right) \mathrm{d} n}_{(h)} \underbrace{\frac{\varepsilon d}{2} \int_{-\infty}^{+\infty}\left[\boldsymbol{t}_{1} \frac{\partial}{\partial s_{1}^{(1)}}\left(\frac{\partial \psi}{\partial s_{1}^{(1)}}\right)^{2}+\boldsymbol{t}_{2} \frac{\partial}{\partial s_{2}^{(1)}}\left(\frac{\partial \psi}{\partial s_{2}^{(1)}}\right)^{2}\right] \mathrm{d} n}_{(i)} \\
& \underbrace{+\varepsilon d\left(\kappa_{1}+\kappa_{2}\right) \int_{-\infty}^{+\infty}\left(\boldsymbol{t}_{1} \frac{\partial \psi}{\partial s_{1}^{(1)}}+\boldsymbol{t}_{2} \frac{\partial \psi}{\partial s_{2}^{(1)}}\right)\left(\frac{\partial \psi}{\partial n^{(1)}}\right) \mathrm{d} n}_{(j)} \\
& \underbrace{-\frac{\varepsilon d}{2} \int_{-\infty}^{+\infty}\left[\boldsymbol{t}_{1} \frac{\partial}{\partial s_{1}^{(1)}}\left(\frac{\partial \psi}{\partial s_{2}^{(1)}}\right)^{2}+\boldsymbol{t}_{2} \frac{\partial}{\partial s_{2}^{(1)}}\left(\frac{\partial \psi}{\partial s_{1}^{(1)}}\right)^{2}\right] \mathrm{d} n}_{(l)} \\
& \underbrace{-\varepsilon^{2} \rho \boldsymbol{n}_{\mathrm{G}} \int_{-\infty}^{\infty} \frac{\partial \mu}{\partial n^{(2)}} \mathrm{d} n}_{(m)} \underbrace{+\varepsilon d \int_{-\infty}^{+\infty}\left[\boldsymbol{t}_{1} \frac{\partial}{\partial s_{2}^{(1)}}\left(\frac{\partial \psi}{\partial s_{1}^{(1)}} \frac{\partial \psi}{\partial s_{2}^{(1)}}\right)+\boldsymbol{t}_{2} \frac{\partial}{\partial s_{1}^{(1)}}\left(\frac{\partial \psi}{\partial s_{1}^{(1)}} \frac{\partial \psi}{\partial s_{2}^{(1)}}\right)\right] \mathrm{d} n}_{(k)}
\end{aligned}
$$

Here, for the sake of simplicity, we focus on the terms (a), (b), (c), and (m) (this is new term.) as follows:

$$
0=\left(P_{\mathrm{G}}-P_{\mathrm{L}}\right) \boldsymbol{n}_{\mathrm{G}}-\boldsymbol{n}_{\mathrm{G}} \varepsilon\left(\kappa_{1}+\kappa_{2}\right) \sigma-\varepsilon^{2} \rho \boldsymbol{n}_{\mathrm{G}} \int_{-\infty}^{\infty} \frac{\partial \mu}{\partial n^{(2)}} \mathrm{d} n
$$

In this equation, the third term is transformed into

$$
\int_{-\infty}^{\infty} \frac{\partial \mu}{\partial n^{(2)}} \mathrm{d} n=\int_{-\infty}^{\infty} \frac{\partial \mu}{\partial P} \frac{\partial P}{\partial n^{(2)}} \mathrm{d} n+\int_{-\infty}^{\infty} \frac{\partial \mu}{\partial T} \frac{\partial T}{\partial n^{(2)}} \mathrm{d} n
$$

In the present discussion, change in temperature is not considered. Thus, the second term on the right-hand side of Eq. (43) is omitted. Here, the thermodynamic relation is considered as follows:

$$
\mathrm{d} \mu_{i}=-\overline{s_{i}} \mathrm{~d} T+\bar{V}_{i} \mathrm{~d} P+\sum_{j}^{N_{j}} \mu_{i j}^{c} \mathrm{~d} n_{j}
$$




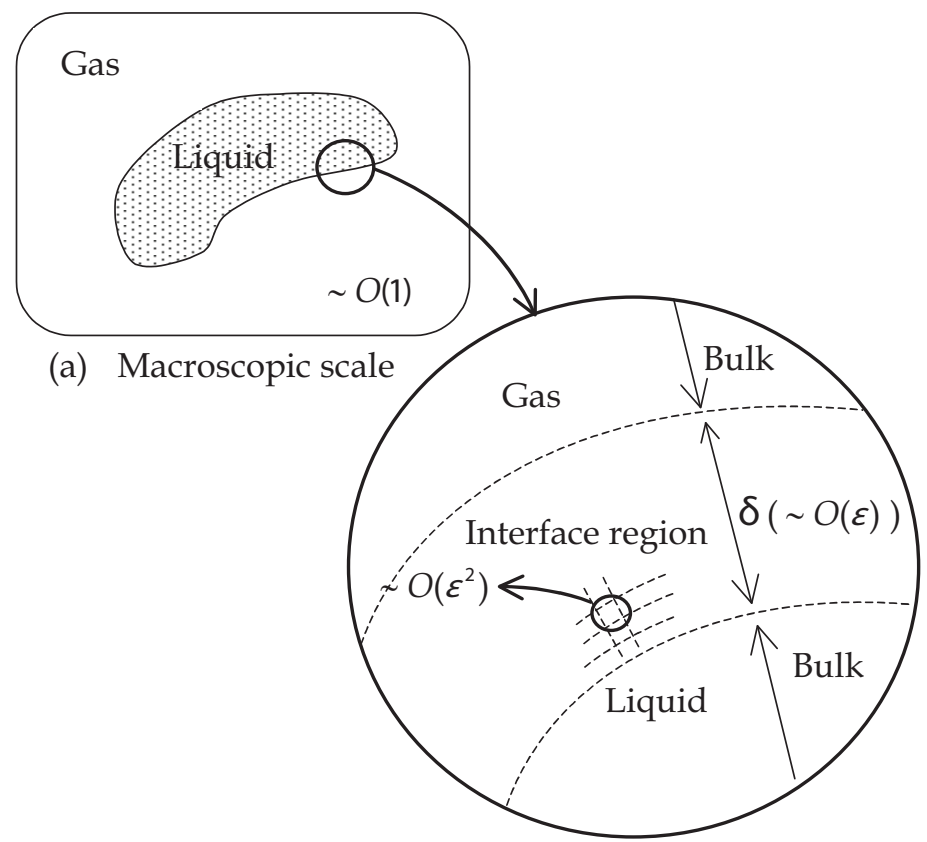

(a) Mesoscopic scale

Fig. 9. Multi-scale schematic around the interface region

In this equation, $\bar{s}_{i}$ and $\bar{V}_{i}$ are the enthalpy and volume per unit mol of component $i$. In the derivation, adding or reducing new components from the external system is not considered. Thus, $\mathrm{d} n_{j}=0$. The following equation is derived from Eq. (44) under constant temperature.

$$
\begin{aligned}
\left(\frac{\partial \mu_{i}}{\partial P}\right)_{T, n_{j}} & =\bar{V}_{i} \\
& =\frac{\mathrm{R} T}{P_{i}}
\end{aligned}
$$

Then, the substitution of Eq. (45) into the third term in Eq. (42) by considering Eq. (43) and transformation of the equation yields

$$
\begin{aligned}
\int_{-\infty}^{\infty} \frac{\partial \mu}{\partial n^{(2)}} \mathrm{d} n & =\int_{-\infty}^{\infty} \frac{\partial \mu}{\partial P} \frac{\partial P}{\partial n^{(2)}} \mathrm{d} n \\
& =\int_{-\infty}^{\infty} \frac{\partial \mu}{\partial P} \mathrm{~d} P \\
& =\int_{P_{i \mathrm{G}}}^{P_{\mathrm{iL}}} \frac{\mathrm{R} T}{P_{i}} \mathrm{~d} P \\
& =\mathrm{R} T \ln \left(\frac{P_{i \mathrm{~L}}}{P_{i \mathrm{G}}}\right)
\end{aligned}
$$


Then, the following equation is obtained by considering Eqs. (46) and (42).

$$
0=\left(P_{\mathrm{G}}-P_{\mathrm{L}}\right) \boldsymbol{n}_{\mathrm{G}}-\boldsymbol{n}_{\mathrm{G}} \varepsilon\left(\kappa_{1}+\kappa_{2}\right) \sigma-\varepsilon^{2} \rho \boldsymbol{n}_{\mathrm{G}} \mathrm{R} T \ln \left(\frac{P_{i \mathrm{~L}}}{P_{i \mathrm{G}}}\right)
$$

In this equation, $P_{\mathrm{L}}$ and $P_{\mathrm{G}}$ are the pressure in bulk phase. The Kelvin equation explains the difference in vapor pressure between the flat surface $(H=0)$ and curved surface ( $H \neq 0)$. The mean curvature $H=0$ in Eq. (47) gives

$$
0=\left(P_{\mathrm{G}}-P_{\mathrm{L}}\right) \boldsymbol{n}_{\mathrm{G}}-\varepsilon^{2} \rho \boldsymbol{n}_{\mathrm{G}} \mathrm{R} T \ln \left(\frac{P_{i 0 \mathrm{~L}}}{P_{i 0 \mathrm{G}}}\right)
$$

Thus, the subtraction of Eq. (48) from Eq. (47) is

$$
0=\boldsymbol{n}_{\mathrm{G}} \varepsilon\left(\kappa_{1}+\kappa_{2}\right) \sigma-\varepsilon^{2} \rho \boldsymbol{n}_{\mathrm{G}} \mathrm{R} T \ln \left(\frac{P_{i \mathrm{~L}}}{P_{i \mathrm{G}}} \frac{P_{i 0 \mathrm{G}}}{P_{i 0 \mathrm{~L}}}\right)
$$

In Eq. (49), we assume $P_{i \mathrm{G}} \approx P_{i 0 \mathrm{G}}$ and focus on the liquid phase. Equation (49) becomes

$$
0=\boldsymbol{n}_{\mathrm{G}} \varepsilon\left(\kappa_{1}+\kappa_{2}\right) \sigma-\varepsilon^{2} \rho \boldsymbol{n}_{\mathrm{G}} \mathrm{R} T \ln \left(\frac{P_{i \mathrm{~L}}}{P_{i 0 \mathrm{~L}}}\right)
$$

Considering the equilibrium state in Eq. (50), the coefficient of the normal vector is set to zero. Finally, the following equation is obtained.

$$
\frac{2 H \sigma V_{m}}{\mathrm{R} T}=\varepsilon \ln \left(\frac{P_{i \mathrm{~L}}}{P_{i 0 \mathrm{~L}}}\right)
$$

where $V_{m}=1 / \rho$. This equation is the Kelvin equation derived from the multi-scale multiphase flow equation.

\section{Conclusion}

In a previous study, a new interfacial model of the gas-liquid interface was developed based on thermodynamics, assuming that the interface has a finite thickness, similar to a thin fluid membrane. In particular, the free energy was derived based on a lattice-gas model that includes the electrostatic potential due to contamination. The free energy was incorporated into the NS equation by using the Chapman-Enskog expansion. Finally, a multi-scale multiphase flow equation was derived that characterizes the mesoscopic scale. The interfacial equation for a macroscopic-scale gas-liquid interface is characterized by a jump condition. In the present study, the jump condition at the gas-liquid interface treated by thermodynamics was derived by using the multi-scale multiphase flow equation and compared with the conventional jump condition. Finally, we developed the multi-scale gasliquid interfacial model; this model supports the interfacial phenomena from the microscopic to macroscopic scale theoretically to give the following results:

1. The thermodynamic interfacial jump condition was derived by using the multi-scale multiphase flow equation. The present study indicated the relationship between the 
mechanical and thermodynamic approaches with respect to the model of the gas-liquid interface.

2. The Marangoni-effect terms, which are related to temperature differences and contamination at the interface, were included in some additional terms derived under the thermodynamic jump condition.

3. From the normalized thermodynamic jump condition, we obtained a new dimensionless number $\mathrm{N}$ that represents the relationship between the electrostatic force due to contamination at the interface and the hydrodynamic force. The order estimation of $\mathrm{N}$ suggests that we may be able to specifically classify bubble coalescence or breakup.

4. Considering term (d) of Eq. (23), we concluded that the conventional jump condition holds true for Eqs. (20) and (30). Therefore, the conventional jump condition is restricted to the case of spherical bubbles or droplets and is inaccurate when the relationship between the surface tensions in the $s_{1}$ and $s_{2}$ tangential directions is imbalanced.

5. On the basis of the multi-scale multiphase flow equation, we derived the Kelvin equation. This result indicates that equation (37) contains the physics for the evaporation/condensation of a curved surface and will support other interfacial phenomena characterized by thermodynamics. However, more detailed discussion of Eq. (51) is needed because other terms in Eq. (41) are omitted in the derivation of Eq. (51).

In our recent study (Yonemoto \& Kunugi, 2010c), the momentum jump condition was applied to a discussion on an equilibrium condition at a three-phase contact line of a sessile droplet on a smooth solid surface. Furthermore, the equilibrium condition of the sessile droplet was also considered by the thermodynamic approach (Yonemoto \& Kunugi, 2009). Therefore, our new interfacial model may allow the development of a general multi-scale interfacial model that can treat two-phase and three-phase interfaces theoretically in the future.

\section{References}

Álvarez, E., Sanjurjo, B., Cancela, A. \& Navaza, J. M. (2000). Mass transfer and influence of solutions in a bubbling column, Chem. Eng. Res. Des., Vol. 78(6), September 2000, pp. 889-893

Anderson, D. M., McFadden, G. B. \& Wheeler, A. A. (1998). Diffuse-interface methods in fluid mechanics, Annu. Rev. Fluid Mech., Vol. 30, January 1998, pp. 139-165, ISSN 0066-4189

Aris, R. (1962). Vectors, tensors and the basic equations of fluid mechanics, ISBN 0486661105, Prentice-Hall

Butt, H-J, Graf, K. \& Kappl, M. (2003). Physics and chemistry of interfaces, ISBN 3527404139, WILEY-VCH Verlag GmbH \& Co. KgaA, Weinheim

Cahn, J. W., Hilliard, J. E. (1958). Free energy of a nonuniform system, I. Interfacial energy, J. Chem. Phys., Vol. 28, No. 2, February 1958, pp. 258-267, ISSN 1089-7690

Chapman, S., Cowling, T. (1970). The mathematical theory of non-uniform gases, 3rd ed. ISBN 9780521408448, Cambridge University Press 
Craig, V. S. J. (2004). Bubble coalescence and specific-ion effects, Curr Opin Colloid Interface Sci., Vol. 9, August 2004, pp. 178-184, ISSN 1359-0294

Delhaye, J. M. (1974). Jump conditions and entropy surfaces in two-phase systems. Local instant formulation, Int. J. Multiphase Flow, Vol. 1, June 1974, pp. 395-409, ISSN 0301-9322

Fialkowski, M., Aksimentiev, A. \& Holyst, R. (2001). Scaling of the euler characteristic, surface area, and curvatures in the phase separating or ordering systems, Phys. Rev. Lett., Vol. 86, No. 2, January 2001, pp. 240-243, ISSN 1079-7114

Graciaa, A., Morel, G., Paulner, P., Lachaise, J. \& Schechter, R. S. (1995). The $\zeta$-potential of gas bubbles, J. Colloid Interface Sci., Vol. 172, June 1995, pp. 131-136, ISSN 0021-9797

Henry, C. L., Craig, V. S. J. (2008). Ion-specific influence of electrolytes on bubble coalescence in nonaqueous solvents, Langmuir, Vol. 24, July 2008, pp. 7979-7985, ISSN 1520-5827

Hong, W. H. \& Brauer, H. (1989). Gas-liquid mass transfer in bubble - column reactors, Int. Chem. Eng., Vol. 29(3), July 1989, pp. 388-434

Lessaard, R. R. \& Zieminski, S. A. (1971). Bubble coalescence and gas transfer in aqueous electrolytic solutions, Ind. Eng. Chem. Fundam., Vol. 10, No. 2, May 1971, pp. $260-$ 269, ISSN 0196-4313

Marčelja, S. (2004). Short-range forces in surface and bubble interaction, Curr Opin Colloid Interface Sci., Vol. 9, August 2004, pp. 165-167, ISSN 1359-0294

Onsager, L. (1931a). Reciprocal relations in irreversible processes. I., Phys. Rev., Vol. 37, February 1931a, pp. 405-426, ISSN 1536-6065

Onsager, L. (1931b). Reciprocal relations in irreversible processes. II., Phys. Rev., Vol. 38, December 1931b, pp. 2265-2279, ISSN 1536-6065

Ribeiro Jr, C. P., Mewes, D. (2007). The influence of electrolytes on gas hold-up and regime transition in bubble columns, Chem. Eng. Sci., Vol. 62, September 2007, pp. 45014509, ISSN 0009-2509

Rowlinson, J. S., Widom, B. (1984), Molecular theory of capillarity, Oxford University Press

Safran, S. A. (1994). Statistical thermodynamics of surfaces, interfaces, and membranes, ISBN 9780813340791, ADDISON-WESLEY PUBLISHING COMPANY

Scriven, L. E. (1960). Dynamics of a fluid interface: equation of motion for Newtonian surface fluids, Chem. Eng. Sci., Vol. 12, May 1960, pp. 98-108, ISSN 0009-2509

Tsang, T. H., Koh, Y-H. \& Koch, D. L. (2004). Bubble-size dependence of the critical electrolyte concentration for inhibition of coalescence, J. Colloid Interface Sci., Vol. 275, July 2004, pp. 290-297, ISSN 0021-9797

Yonemoto, Y., Kunugi, T. \& Serizawa, A. (2005). Study on bubble shape interacted with vortex motion via mathematical approach, 58th Annual Meeting of the Division of Fluid Dynamics, Vol. 50, No. 9, November 2005, p 250

Yonemoto, Y., Yanagisawa, H., Kawara, Z. \& Kunugi, T. (2008). Coalescence of microbubble, J. JSEM, Vol. 8, No. 1, March 2008, pp. 38-44, ISSN 13464930

Yonemoto, Y., Kunugi, T. (2009). Discussion on a mechanical equilibrium condition of a sessile drop on a smooth solid surface, J. Chem. Phys., Vol. 130, No. 14, April 2009, pp. 144106-1-144106-12, ISSN 1089-7690 
Yonemoto. Y, Kunugi, T. (2010a). Multi-scale modeling of the gas-liquid interface based on mathematical and thermodynamic approaches, Open transport phenom.J., Vol. 2, pp. 69-79

Yonemoto, Y., Kunugi, T. (2010b). Fundamental numerical simulation of microbubble interaction using multi-scale multiphase flow equation, Microgravity Sci. Technol., Vol. 22, September 2010b, pp. 397-405, ISSN 1875-0494

Yonemoto, Y, Kunugi, T. (2010c). Macroscopic wettability based on an interfacial jump condition, Phys. Rev. E., Vol. 81, May 2010c, pp. 056310-1-056310-8, ISSN 1550-2376 


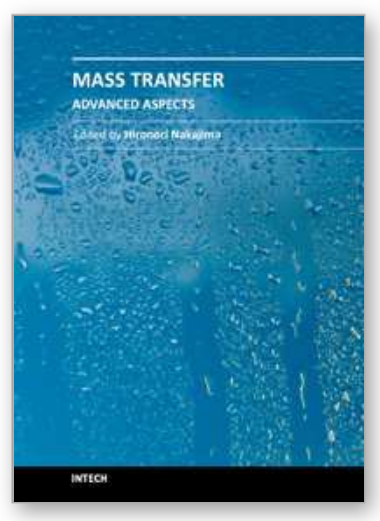

\author{
Mass Transfer - Advanced Aspects \\ Edited by Dr. Hironori Nakajima
}

ISBN 978-953-307-636-2

Hard cover, 824 pages

Publisher InTech

Published online 07, July, 2011

Published in print edition July, 2011

Our knowledge of mass transfer processes has been extended and applied to various fields of science and engineering including industrial and manufacturing processes in recent years. Since mass transfer is a primordial phenomenon, it plays a key role in the scientific researches and fields of mechanical, energy, environmental, materials, bio, and chemical engineering. In this book, energetic authors provide present advances in scientific findings and technologies, and develop new theoretical models concerning mass transfer. This book brings valuable references for researchers and engineers working in the variety of mass transfer sciences and related fields. Since the constitutive topics cover the advances in broad research areas, the topics will be mutually stimulus and informative to the researchers and engineers in different areas.

\title{
How to reference
}

In order to correctly reference this scholarly work, feel free to copy and paste the following:

Yukihiro Yonemoto and Tomoaki Kunugi (2011). Macroscopic Gas-Liquid Interfacial Equation Based on Thermodynamic and Mathematical Approaches, Mass Transfer - Advanced Aspects, Dr. Hironori Nakajima (Ed.), ISBN: 978-953-307-636-2, InTech, Available from: http://www.intechopen.com/books/mass-transferadvanced-aspects/macroscopic-gas-liquid-interfacial-equation-based-on-thermodynamic-and-mathematicalapproaches

\section{INTECH}

open science | open minds

\author{
InTech Europe \\ University Campus STeP Ri \\ Slavka Krautzeka 83/A \\ 51000 Rijeka, Croatia \\ Phone: +385 (51) 770447 \\ Fax: +385 (51) 686166 \\ www.intechopen.com
}

\author{
InTech China \\ Unit 405, Office Block, Hotel Equatorial Shanghai \\ No.65, Yan An Road (West), Shanghai, 200040, China \\ 中国上海市延安西路65号上海国际贵都大饭店办公楼 405 单元 \\ Phone: +86-21-62489820 \\ Fax: $+86-21-62489821$
}


(C) 2011 The Author(s). Licensee IntechOpen. This is an open access article distributed under the terms of the Creative Commons Attribution 3.0 License, which permits unrestricted use, distribution, and reproduction in any medium, provided the original work is properly cited. 